\title{
Single-Point Left Ventricular Pacing Optimized by ECG Results in Better Resynchronization than Multipoint Pacing
}

\author{
Rodolfo San Antonio ${ }^{1}$, Eduard Guasch ${ }^{1}$, Ana González-Ascaso ${ }^{2}$, Rafael Jiménez-Arjona ${ }^{1}$, \\ Andreu Climent ${ }^{2}$, Margarida Pujol-Lopez ${ }^{1}$, Adelina Doltra ${ }^{1}$, Francisco Alarcón ${ }^{1}$, Paz \\ Garre $^{1}$, Alejandro Liberos ${ }^{2}$, Omar Trotta $^{1}$, Levio Quinto ${ }^{1}$, Roger Borràs ${ }^{3}$, Elena Arbelo ${ }^{4}$, \\ Ivo Roca-Luque ${ }^{1}$, Felipe Atienza ${ }^{5}$, Josep Brugada ${ }^{6}$, Francisco Fernández-Avilés ${ }^{5}$, María \\ Guillem $^{2}$, Marta Sitges ${ }^{1}$, Jose Maria Tolosana ${ }^{4}$, and Luis Mont Girbau ${ }^{1}$ \\ ${ }^{1}$ Hospital Clinic de Barcelona \\ ${ }^{2}$ Universitat Politecnica de Valencia \\ ${ }^{3}$ Hospital Clínic \\ ${ }^{4}$ Hospital Clinic, Universitat de Barcelona; \\ ${ }^{5}$ Gregorio Maranon General University Hospital Cardiology Service \\ ${ }^{6}$ Hospital Clinic
}

October 5, 2020

\begin{abstract}
Background: Multipoint pacing (MPP) in cardiac resynchronization therapy (CRT) activates the left ventricle from two locations, thereby shortening the QRS duration and enabling better resynchronization; however, compared with conventional CRT, MPP reduces battery longevity. On the other hand, electrocardiogram-based optimization using the fusion-optimized intervals (FOI) method achieves more significant reverse remodeling than nominal CRT programming. Our study aimed to determine whether MPP could attain better resynchronization than single-point pacing (SPP) optimized by FOI. Methods: This prospective study included 32 consecutive patients who successfully received CRT devices with MPP capabilities. After implantation, the QRS duration was measured during intrinsic rhythm and with three pacing configurations: MPP, SPP-FOI, and MPP-FOI. In 14 patients, biventricular activation times (by electrocardiographic imaging, ECGI) were obtained during intrinsic rhythm and for each pacing configuration to validate the findings. Device battery longevity was estimated at the 45-day follow-up. Results: The SPP-FOI method achieved greater QRS shortening than MPP (-56 \pm 16 vs. $-42 \pm 17$ ms, P $<0.001)$. Adding MPP to the best FOI programming did not result in further shortening (MPP-FOI: $-58 \pm 14 \mathrm{~ms}, \mathrm{P}=0.69$ ). Furthermore, the biventricular activation time according to ECGI was also shorter with SPP-FOI than with MPP. The estimated battery longevity was longer with SPP than with MPP ( $8.1 \pm 2.3$ vs. $6.3 \pm 2.0$ years, $\mathrm{P}=0.03)$. Conclusions: SPP optimized by FOI resulted in better resynchronization and longer battery duration than MPP.
\end{abstract}

\section{Introduction}

Cardiac resynchronization therapy (CRT) with multipoint pacing (MPP) shortens the QRS duration ${ }^{1}$ and improves outcomes compared to single-point left ventricular (LV) pacing. ${ }^{2,3}$ However, MPP causes higher battery drain. ${ }^{4-6}$ On the other hand, electrocardiographic optimization using the fusion-optimized intervals (FOI) method also shortens the QRS duration ${ }^{7-9}$ and achieves greater LV reverse remodeling than nominal programming. ${ }^{10}$

The magnitude of QRS shortening is associated with clinical and prognostic improvements in patients with $\mathrm{CRT}^{11}$ and serves as a surrogate for the cardiac activation time. The activation time can be measured 
through electrocardiographic imaging (ECGI) using body surface potential mapping. Thus, ECGI yields a high-resolution single-beat ventricular activation map. ${ }^{12}$

The objective of the present study was to determine whether MPP could attain better resynchronization than single-point pacing (SPP) optimized by FOI.

\section{Methods}

\section{Study population}

The inclusion criteria were as follows: New York Heart Association functional class II to III, LV ejection fraction (LVEF) of $35 \%$ or less, prolonged QRS width ([?]150 ms), and sinus rhythm with intact atrioventricular (AV) conduction (PR interval [?] $250 \mathrm{~ms}$ ). The exclusion criteria were persistent or permanent atrial fibrillation, recent (within 40 days) myocardial infarction, cardiac surgery, or coronary revascularization within three months or scheduled within the following 12 months, intravenous inotropic support within 30 days, and planned heart transplantation within the next 12 months. We prospectively enrolled 32 consecutive patients fulfilling the current class I/IIa indications for the implantation of a CRT device. ${ }^{13}$

\section{Study protocol}

All included patients underwent implantation of a CRT device and were tested for each of the 3 pacing configurations (MPP, SPP-FOI, MPP-FOI). The study protocol is summarized in Figure 1 . Written consent was obtained for each patient. The protocol was reviewed and approved by the local institutional research ethics committee (HCB/2018/0388).

a) CRT device implantation

All patients underwent CRT device implantation according to routine clinical practice. Each patient was implanted with a commercially available MPP-capable CRT system that included a quadripolar LV lead and a compatible CRT device. The indications for pacemakers or defibrillators were based on comorbidities and clinical factors limiting the quality of life or life expectancy. The atrial lead was positioned in the right atrial appendage, and the right ventricular (RV) lead was positioned in the RV apical septum. The LV quadripolar leads (four electrodes: D1, M2, M3, and P4 from distal to proximal) preferentially targeted a lateral or posterolateral branch of the coronary sinus. Positions were defined using radiological criteria and checked in 30-degree right and left anterior oblique projections immediately after implantation using fluoroscopy and the next day using X-ray.

b) Electrocardiographic optimization

Device optimization was performed immediately after implantation in the operating room. The QRS duration was measured during intrinsic rhythm and with the three specific configurations: MPP, SPP-FOI, and MPP-FOI. The 12 leads of a surface electrocardiogram (ECG) were simultaneously displayed in vertical alignment and digitally recorded for off-line measurement (WorkMate Claris; St. Jude Medical, Abbott). Time measurements were taken at a screen velocity of $200 \mathrm{~mm} / \mathrm{s}$ by two experienced observers, unaware of the configurations, using a digital cursor with an accuracy of $1 \mathrm{~ms}$; the mean across 3 consecutive cycles was calculated and recorded. The onset of QRS was considered to be the start of the fast deflection ${ }^{9}$ to avoid the average 45-ms transmural delay due to epicardial pacing of the LV lead. At discharge, the device was programmed with the configuration that provided the narrowest QRS duration.

Multipoint pacing programming: Multipoint pacing was programmed as previously described. ${ }^{3,4,14}$ The MPP configuration was chosen to first pace using the distal electrode (primarily D1, secondarily M2) as the cathode (LV1) and then the proximal electrode (primarily P4, secondarily M3) as the cathode (LV2). Two LV vectors with wide interelectrode cathode spacing were selected as the final LV1/LV2 vectors, utilizing the following order of preference: D1/P4 $>$ D1/M3 or M2/P4 (Figure S1 ). When the LV pacing vector showed a capture threshold larger than $3.5 \mathrm{~V}$ and/or phrenic nerve stimulation (PNS), it was excluded. Programming included the following intervals: (1) fixed AV interval, $130 \mathrm{~ms}$; (2) RV-LV2 ([?]1) interval, $5 \mathrm{~ms}$; and (3) LV1-LV2 ([?]2) interval, $5 \mathrm{~ms}$ ([?]1 and [?]2 with the minimal programmable delay). 
Fusion-optimized intervals method: FOI optimization was performed as described elsewhere. ${ }^{7,10}$ Briefly, the fusion band was determined with atrial sensing and ventricular pacing with the anatomically furthest electrode in the LV lead. Pacing was initially tested with the longest AV interval that allowed LV capture; the AV interval was subsequently shortened in 20-ms decrements until LV-only capture was achieved. The AV interval that yielded the narrowest QRS was chosen and considered the AV FOI (Figure S2 ). This procedure was repeated during atrial pacing at 10 beats/min above spontaneous sinus rhythm. Afterwards, the interventricular (VV) interval was adjusted by programming the one that achieved the shortest QRS (simultaneous RV and LV pacing [VV $0 \mathrm{~ms}$ ], $30 \mathrm{~ms}$ LV pre-excitation, and $30 \mathrm{~ms}$ RV pre-excitation). These VV intervals were chosen because previous studies indicated that most patients showed the best VVs in this range. ${ }^{7,10}$ The VV value that achieved the narrowest QRS was considered the VV FOI.

Combined MPP-FOI strategy: The AV and [?]1 (RV-LV2) intervals were optimized as in the FOI method. Using the pacing vectors LV1 and LV2 obtained for the MPP strategy, [?]2 was ultimately programmed to $5 \mathrm{~ms}$ (minimum programmable delay).

c) Electrocardiographic imaging

The biventricular activation time was evaluated using ECGI recordings (CORIFY Care SL) obtained while running four programming configurations: (1) intrinsic rhythm, (2) MPP, (3) SPP-FOI, and (4) MPP-FOI.

The torso surface potential was obtained using body surface potential mapping, wherein 64 electrodes attached to the skin were recorded at a sampling rate of $1000 \mathrm{~Hz}$ for off-line processing. Electrode location and torso and heart volumes were determined based on (1) cardiac images acquired via computed tomography or magnetic resonance imaging obtained before device implantation and (2) photogrammetry techniques that enable $3 \mathrm{D}$ reconstruction of the torso from a standard video recording.

An average PQRST interval was calculated for each lead using template matching averaging after removing pacing artifacts. An inverse computed electrogram was reconstructed for the heart's surface by solving the inverse problem using zero-order Tikhonov and L-curve methods. ${ }^{15}$

The biventricular activation time was calculated by (1) a set of sinusoidal wavelets for all time instants with a negative slope within the QRS complex and with an amplitude proportional to the slope at that time and (2) the instantaneous phase of Hilbert's transform of a composite signal of all sinusoidal wavelets. The activation times corresponded to the points where phase inversions occurred in that instantaneous phase. The total biventricular activation time was defined as the difference between the last and first activation times.

d) Echocardiographic measurements

An echocardiography specialist blinded to device programming obtained standard Doppler echocardiographic images using a commercially available system (Vivid 7; General Electric, Milwaukee, WI, USA).

Each patient was evaluated at baseline (device in OFF) and with each pacing configuration (MPP, SPP-FOI, and MPP-FOI). Cardiac asynchrony was evaluated at several levels: (1) left intraventricular asynchrony using septal flash ${ }^{16}$ and $2 \mathrm{D}$ strain, (2) AV asynchrony using the diastolic filling ratio (diastolic filling time/RR interval duration), ${ }^{17}$ and (3) VV asynchrony quantified as the difference between the LV and RV pre-ejection times with pulsed-wave Doppler (Figure S3 ). ${ }^{18}$ In the 2D strain analysis, the time difference between the peak systolic strain at opposing LV walls (mid-septal and mid-lateral) was measured. Finally, the LV outflow tract velocity time integral, a measure of cardiac systolic function, was quantified.

e) Estimated battery longevity

At 45 days post-implantation, data for estimating battery longevity with the final programmed configuration (MPP, SPP-FOI, or MPP-FOI) were collected, and estimated battery life was compared between patients who had MPP and SPP activated. Right atrial and RV pacing outputs were programmed at $2.5 \mathrm{~V} / 0.5 \mathrm{~ms}$, and LV pacing output was programmed at 1.5 times the pacing threshold. 


\section{Statistical analysis}

We estimated that 32 patients would be needed to find a 10-ms difference in the QRS duration between MPP and MPP-FOI with an $\alpha=0.05$ and a statistical power $(1-\beta)$ of $80 \%$, assuming a standard deviation of $20 \mathrm{~ms}$ for pair differences.

Categorical variables, represented as proportions, were compared using the $\chi^{2}$ test. Continuous variables, after confirming their normal distribution (QQ plot), were expressed as the means and standard deviations, and between-group comparisons were made using Student'st -test. Linear mixed-effects models with a repeated-measures structure were used to assess differences in the QRS duration, echocardiographic measures, and biventricular activation times (using ECGI) across the three pacing modes. The correlation between the duration of the QRS complex (ECG) and activation time (ECGI) was assessed using the Pearson coefficient. A two-tailed $P<0.05$ was deemed statistically significant. All statistical analyses were conducted using SPSS software v22.0 (SPSS Inc., USA) or R v3.6.1 (R Project for Statistical Computing; Vienna, Austria).

\section{Results}

From May 2018 through February 2020, 32 consecutive patients were prospectively recruited. No patients were lost during the 45-day follow-up. The primary patient characteristics are shown inTable 1 .

\section{CRT device implantation}

Twenty-six patients (81\%) received a CRT defibrillator; all others received a CRT pacemaker. The devices were produced by one of two manufacturers: Abbott (20,62\%) or Boston Scientific (12, 38\%). The quadripolar LV lead was placed transvenously in a coronary vein overlying the LV free wall: 14 (44\%) in a lateral vein and $18(56 \%)$ in a posterolateral vein.

\section{Electrocardiographic optimization}

Measurements of the QRS duration during intrinsic rhythm and with the three pacing configurations were recorded in the 32 patients. The results are plotted in Figure 2 . Compared to MPP, SPP-FOI and MPPFOI achieved greater QRS shortening: the $\Delta$ QRS with SPP-FOI was $-56 \pm 16$ ms; with MPP was $-42 \pm 17$ ms; and with MPP-FOI was $-58 \pm 14 \mathrm{~ms}$; omnibus $P<0.001$. The differences in QRS shortening between SPP-FOI and MPP-FOI were not significant ( $P=0.69$, with a difference between groups of $2 \pm 7 \mathrm{~ms}$ ).

The pacing configuration at discharge is summarized in Figure 3 . The shortest QRS duration was achieved using SPP-FOI in 9 patients (28\%), MPP in 1 patient (3\%), and MPP-FOI in 22 patients (69\%). Among the latter two groups combined, 12 out of $23(52 \%)$ patients were not candidates for chronic MPP because of either (1) the pacing thresholds exceeded $3.5 \mathrm{~V} / 0.4 \mathrm{~ms}$ at the distal $(4,17 \%)$ or proximal $(6,26 \%)$ cathodes or (2) incorrigible PNS (2, 9\%).

In the SPP configuration, the left ventricular cathode was D1 in 20 patients (62\%), M2 in 6 (19\%), M3 in 5 $(16 \%)$, and $\mathrm{P} 4$ in $1(3 \%)$. For the MPP configurations, the two selected LV vectors were D1/P4 in 12 patients (37\%), D1/M3 in $14(44 \%)$, and M2/P4 in $6(19 \%)$.

\section{Electrocardiographic imaging}

Noninvasive cardiac mapping was obtained from 14 patients in whom recent ( $<3$ months) cardiac computed tomography or magnetic resonance images were available. Two patients were excluded (one due to atrial fibrillation during mapping and one because of insufficient image quality). As expected, LV activation was delayed in comparison to RV activation at intrinsic rhythm, while CRT activation led to a more synchronous activation pattern in the LV (Figure 4 ). At intrinsic rhythm, the biventricular activation time was $90 \pm 17$ ms. Both FOI configurations achieved the shortest biventricular activation times $(72 \pm 14 \mathrm{~ms}$ and $77 \pm 18 \mathrm{~ms}$ for SPP-FOI and MPP-FOI, respectively), and they were significantly shorter than the activation times during intrinsic rhythm $(\mathrm{P}=0.005$ and $\mathrm{P}=0.04$, respectively). No differences were observed between the activation times during intrinsic rhythm and the MPP strategy alone $(83 \pm 19 \mathrm{~ms}, \mathrm{P}=0.30)$ (Figure 5 ). 
The biventricular activation times according to ECGI showed a good correlation with the QRS durations determined via ECG $(\mathrm{r}=0.58$; Figure 6$)$.

\section{Echocardiographic measures}

An echocardiogram was available in 30 patients. As illustrated inTable $\mathbf{S} 4$, the 3 configurations did not differ in left ventricular outflow tract velocity time integral or in asynchrony parameters.

\section{Estimated battery longevity}

The estimated mean battery longevity was significantly shorter with the MPP strategy (MPP-ON; $n=11$ ) than with the SPP strategy (MPP-OFF; $\mathrm{n}=21$ ), resulting in mean battery longevity estimates of $6.3 \pm 2.0$ years and $8.1 \pm 2.3$ years, respectively $(P=0.03)$. The mean $L V$ pacing output was $2.7 \pm 0.6 \mathrm{~V} / 0.5 \mathrm{~ms}$ with the SPP configuration and was similar to that with the MPP configuration (LV1, 2.5 $\pm 0.7 \mathrm{~V} / 0.5 \mathrm{~ms}$; LV2, $2.8 \pm 0.7$ ). At 45 days post-implantation, the RV and right atrial thresholds and the percentage of atrial pacing and biventricular pacing were similar in both groups. Additionally, no differences were found based on the device type or manufacturer (Table S5 ). No defibrillator shocks occurred among the entire sample during the 45-day follow-up period.

\section{Discussion}

The main finding of the present study is that single-point LV pacing optimized by FOI enabled greater QRS shortening than MPP alone. Moreover, the use of SPP significantly prolonged the expected battery longevity compared with MPP. The addition of MPP to FOI optimization did not result in a significant additional reduction in the QRS duration. Finally, a high percentage of patients were not candidates for MPP because of high pacing thresholds or PNS.

Compared with conventional CRT, MPP via a quadripolar LV lead has demonstrated acute and long-term improvements in hemodynamic, clinical, and echocardiographic responses. ${ }^{2,3,14}$ However, most studies comparing MPP with standard single-point LV pacing neglect AV optimization. ${ }^{2,13}$ As shown in the BEST study, ${ }^{7,10}$ achieving fusion with intrinsic conduction through the FOI method maximized shortening of the QRS duration and, in turn, induced greater reverse LV remodeling upon follow-up. In the present study, an optimized single-point LV pacing strategy using the FOI method bested an MPP strategy programmed with a fixed AV interval of $130 \mathrm{~ms}$, a wide anatomical LV1-LV2 distance, and minimal intraventricular delay (5 $\mathrm{ms}) .{ }^{4,5}$ Our findings are reinforced by a recent study published by O'Donnell et al. ${ }^{19}$ In this study assessing the $\triangle$ QRS duration immediately after implantation, the authors observed how automatic AV delay using SyncAV (SPP-SyncAV) showed a better response than that using MPP alone. Conversely, O'Donnell et al. found that the combination of MPP-SyncAV was slightly better than SPP-SyncAV ( $\triangle$ QRS duration of 6 $\mathrm{ms}$ ). In our study, adding MPP to FOI did not significantly shorten the QRS duration (only $2 \pm 7 \mathrm{~ms}$ ). Nevertheless, approximately $2 / 3$ of the patients obtained the narrowest QRS with the MPP-FOI configuration. However, the lack of differences either in the biventricular activation times or mechanical asynchrony improvements by echo between SPP-FOI and MPP-FOI strategies, together with the high percentage of patients in whom MPP could not be chronically programmed and the faster battery drain, favors SPP programming as the initial approach.

Electrocardiographic imaging is a noninvasive technique capable of accurately detecting changes in activation patterns resulting from device optimization strategies. ${ }^{20}$ Ploux et al. found that the mean total activation time decreased in CRT responders regarding intrinsic rhythm, while it increased in nonresponders. ${ }^{20}$ In our study, a significant shortening of biventricular activation times (according to ECGI) was achieved with both FOI configurations (SPP-FOI and MPP-FOI) compared with non-paced rhythm. In addition, biventricular activation times correlated well with QRS duration. Therefore, both the ECG and noninvasive cardiac mapping data support the FOI method's ability to obtain the shortest QRS duration and activation time, which are often associated with a better patient outcome. ${ }^{10,11,20}$

Two limitations warrant mentioning regarding MPP. First, the inability to program two LV vectors due to high pacing thresholds or PNS may be a relevant issue in a real-life setting. The MORE-CRT MPP- 
PHASE I study reported high MPP activation rates (approximately 97\%). However, in that study, maximal anatomical separation between LV1 and LV2 was not sought, and the upper limit threshold was left to the physician's discretion. ${ }^{4}$ In our study, the inability to use MPP chronically was very high (12/23 patients, $52 \%)$. We believe that these data, obtained using wide LV1-LV2 anatomical separation and low thresholds ([?] $3.5 \mathrm{~V}$, as recommended), ${ }^{4,6}$ more closely reflect a real-life scenario. New studies evaluating MPP in this context could clarify this issue. Second, MPP programming with high-output pacing can significantly reduce battery longevity, ${ }^{5,6}$ and the consequent need for device replacement is associated with potentially severe complications and carries an economic impact. ${ }^{21}$ This study is the first to assess battery life in patients with MPP activation, low pacing thresholds ([?] 3.5 V), and the D1/P4, D1/M3, and M2/P4 configurations only. We observed a significant difference in the estimated mean battery longevity of the MPP and single-point LV pacing devices of almost 2 years.

The clinical implications of our results are straightforward. Our data suggest that ECG-based optimization by FOI should be the preferable approach in patients undergoing CRT. The addition of MPP should be individualized and carefully evaluated since it may result in greater battery drainage without apparent clinical benefit.

\section{Limitations}

This study has some limitations, including its single-center design, limited sample size, and short-term focus. Additionally, two devices from two manufacturers were used, and as such, the results might not be generalizable to other devices. Finally, all patients included in the present study were in sinus rhythm with PR intervals under $250 \mathrm{~ms}$; therefore, the results do not apply to all patients receiving CRT.

\section{Conclusions}

In CRT, FOI-based optimization results in a greater shortening of the QRS than MPP. The use of MPP would result in a shorter battery longevity, but no benefit over FOI would be gained.

\section{Acknowledgments}

We are indebted to Neus Portella and Carolina Sanroman for secretarial assistance. We also thank Miriam Sanchez, Silvia Vidorreta and Silvia Salinas for their help in data acquisition.

\section{REFERENCES}

1. Pappone C, Ćalović Ž, Vicedomini G, Cuko A, McSpadden LC, Ryu K, Jordan CD, Romano E, Baldi M, Saviano M, Pappone A, Vitale R, Catalano C, Ciaccio C, Giannelli L, Ionescu B, Petretta A, Fragakis N, Fundaliotis A, Tavazzi L, Santinelli V. Improving cardiac resynchronization therapy response with multipoint left ventricular pacing: Twelve-month follow-up study. Heart Rhythm 2015 Jun;12(6):1250-1258.

2. Pappone C, Ćalović Ž, Vicedomini G, Cuko A, McSpadden LC, Ryu K, Romano E, Saviano M, Baldi M, Pappone A, Ciaccio C, Giannelli L, Ionescu B, Petretta A, Vitale R, Fundaliotis A, Tavazzi L, Santinelli V. Multipoint left ventricular pacing improves acute hemodynamic response assessed with pressure-volume loops in cardiac resynchronization therapy patients. Heart Rhythm 2014 Mar;11(3):394-401.

3. Zanon F, Marcantoni L, Baracca E, Pastore G, Lanza D, Fraccaro C, Picariello C, Conte L, Aggio S, Roncon L, Pacetta D, Badie N, Noventa F, Prinzen FW. Optimization of left ventricular pacing site plus multipoint pacing improves remodeling and clinical response to cardiac resynchronization therapy at 1 year. Heart Rhythm 2016;13(8):1644-1651.

4. Leclercq C, Burri H, Curnis A, Delnoy PP, Rinaldi CA, Sperzel J, Lee K, Calò L, Vicentini A, Fernandez Concha J, Thibault B. Cardiac resynchronization therapy non-responder to responder conversion rate in the more response to cardiac resynchronization therapy with MultiPoint Pacing (MORE-CRT MPP) study: results from Phase I. Eur Heart J 2019 Sep 14;40(35):2979-2987.

5. Forleo GB, Gasperetti A, Ricciardi D, Curnis A, Bertaglia E, Caló L, Pignalberi C, Calzolari V, Ribatti V, Lavalle C, Potenza D, Tondi L, Natale V, Notarstefano P, Viecca M, Morani G, Biffi M, Giammaria M, 
Zanon F, Santini L. Impact of multipoint pacing on projected battery longevity in cardiac resynchronization therapy. An IRON-MPP study sub-analysis. J Cardiovasc Electrophysiol 2019 Dec 5;30(12):2885-2891.

6. Akerstrom F, Narvaez I, Puchol A, Pachon M, Martin-Sierra C, Rodriguez-Manero M, Rodriguez-Padial L, Arias MA. Estimation of the effects of multipoint pacing on battery longevity in routine clinical practice. Europace 2018 Jul 1;20(7):1161-1167.

7. Arbelo E, Tolosana JM, Trucco E, Penela D, Borras R, Doltra A, Andreu D, Acena M, Berruezo A, Sitges M, Mansour F, Castel A, Matas M, Brugada J, Mont L. Fusion-optimized intervals (FOI): a new method to achieve the narrowest QRS for optimization of the AV and VV intervals in patients undergoing cardiac resynchronization therapy. J Cardiovasc Electrophysiol 2014 Mar;25(3):283-292.

8. Tamborero D, Mont L, Sitges M, Silva E, Berruezo A, Vidal B, Delgado V, Tolosana JM, Godoy M, Castel A, Brugada J. Optimization of the interventricular delay in cardiac resynchronization therapy using the QRS width. Am J Cardiol 2009 Nov 15;104(10):1407-1412.

9. Auricchio A, Prinzen FW. Non-responders to cardiac resynchronization therapy: the magnitude of the problem and the issues. Circ J . 2011;75(3):521-7.

10. Trucco E, Tolosana JM, Arbelo E, Doltra A, Castel MA, Benito E, Borras R, Guasch E, Vidorreta S, Vidal B, Montserrat S, Sitges M, Berruezo A, Brugada J, Mont L. Improvement of Reverse Remodeling Using Electrocardiogram Fusion-Optimized Intervals in Cardiac Resynchronization Therapy: A Randomized Study. JACC Clin Electrophysiol 2018;4(2):181-189.

11. Jastrzebski M, Baranchuk A, Fijorek K, Kisiel R, Kukla P, Sondej T, Czarnecka D. Cardiac resynchronization therapy-induced acute shortening of QRS duration predicts long-term mortality only in patients with left bundle branch block. Europace 2019 Feb 1;21(2):281-289.

12. Ploux S, Lumens J, Whinnett Z, Montaudon M, Strom M, Ramanathan C, Derval N, Zemmoura A, Denis A, De Guillebon M, Shah A, Hocini M, Jais P, Ritter P, Haissaguerre M, Wilkoff BL, Bordachar P. Noninvasive electrocardiographic mapping to improve patient selection for cardiac resynchronization therapy: beyond QRS duration and left bundle branch block morphology. J Am Coll Cardiol 2013 Jun 18;61(24):24352443.

13. Brignole M, Auricchio A, Baron-Esquivias G, Bordachar P, Boriani G, Breithardt OA, Cleland J, Deharo JC, Delgado V, Elliott PM, Gorenek B, Israel CW, Leclercq C, Linde C, Mont L, Padeletti L, Sutton R, Vardas PE; ESC Committee for Practice Guidelines (CPG). 2013 ESC Guidelines on cardiac pacing and cardiac resynchronization therapy. Eur Heart J 2013 Aug 1;34(29):2281-2329.

14. Zanon F, Baracca E, Pastore G, Marcantoni L, Fraccaro C, Lanza D, Picariello C, Aggio S, Roncon L, Dell'Avvocata F, Rigatelli G, Pacetta D, Noventa F, Prinzen FW. Multipoint pacing by a left ventricular quadripolar lead improves the acute hemodynamic response to CRT compared with conventional biventricular pacing at any site. Heart Rhythm 2015 May;12(5):975-981.

15. Pedron-Torrecilla J, Rodrigo M, Climent AM, Liberos A, Perez-David E, Bermejo J, Arenal A, Millet J, Fernandez-Aviles F, Berenfeld O, Atienza F, Guillem MS. Noninvasive Estimation of Epicardial Dominant High-Frequency Regions During Atrial Fibrillation.J Cardiovasc Electrophysiol. 2016 Apr;27(4):435-42.

16. Sarvari SI, Sitges M, Sanz M, Tolosana JM, Edvardsen T, Stokke TM, Mont L, Bijnens B. Left ventricular dysfunction is related to the presence and extent of a septal flash in patients with right ventricular pacing. Europace 2016 Mar 7;19(2):euw020.

17. van Bommel RJ, Tanaka H, Delgado V, Bertini M, Borleffs CJ, Ajmone Marsan N, Holzmeister J, Ruschitzka F, Schalij MJ, Bax JJ, Gorcsan J 3rd.. Association of intraventricular mechanical dyssynchrony with response to cardiac resynchronization therapy in heart failure patients with a narrow QRS complex. Eur Heart J 2010 Dec;31(24):3054-3062. 
18. Rouleau F, Merheb M, Geffroy S, Berthelot J, Chaleil D, Dupuis JM, Victor J, Geslin P. Echocardiographic Assessment of the Interventricular Delay of Activation and Correlation to the QRS Width in Dilated Cardiomyopathy. Pacing Clin Electrophysiol 2001 Oct;24(10):1500-1506.

19. O’Donnell D, Wisnoskey B, Badie N, Odgers L, Smart T, Ord M, Lin T, Mangual JO, Cranke G, McSpadden LC, Ryu K, Bianchi V, D'Onofrio A, Pappone C, Calo L, Chow A, Betts TR, Thibault B, Varma N. Electrical synchronization achieved by multipoint pacing combined with dynamic atrioventricular delay. J Interv Card Electrophysiol. 2020 Aug 1.

20. Ploux S, Eschalier R, Whinnett ZI, Lumens J, Derval N, Sacher F, Hocini M, Jais P, Dubois R, Ritter P, Haissaguerre M, Wilkoff BL, Francis DP, Bordachar P. Electrical dyssynchrony induced by biventricular pacing: implications for patient selection and therapy improvement. Heart Rhythm. 2015 Apr;12(4):782-91.

21. Poole JE, Gleva MJ, Mela T, Chung MK, Uslan DZ, Borge R, Gottipaty V, Shinn T, Dan D, Feldman LA, Seide H, Winston SA, Gallagher JJ, Langberg JJ, Mitchell K, Holcomb R, REPLACE Registry Investigators. Complication Rates Associated With Pacemaker or Implantable Cardioverter-Defibrillator Generator Replacements and Upgrade Procedures. Circulation 2010 Oct 19 122(16):1553-1561.

\section{Figure Legends}

Figure 1 Study protocol. AF, atrial fibrillation; CRT, cardiac resynchronization therapy; ECG, electrocardiography; ECGI, electrocardiographic imaging; FOI, fusion-optimized intervals; LVEF, left ventricular ejection fraction; MPP, multipoint pacing; NYHA, New York Heart Association; SPP, single-point pacing.

Figure 2 Box plot showing the $\triangle$ QRS duration (QRS shortening compared with baseline QRS) at each of the three pacing configurations: SPP-FOI, MPP, and SPP-FOI+MPP. The median $\triangle \mathrm{QRS}$ duration is indicated by the black line inside the box. FOI, fusion-optimized intervals; MPP, multipoint pacing; SPP, single-point pacing.

Figure 3 Programming at discharge with the shortest possible QRS duration. FOI, fusion-optimized intervals; MPP, multipoint pacing.

Figure 4 ECGI during the biventricular activation sequence in a patient with left bundle branch block. The upper panel represents an anterior view; the inferior panel represents a posterior view. At baseline, cardiac activation starts from the RV, but in pacing configurations, cardiac activation changes to simultaneous RVLV. FOI, fusion-optimized intervals; LV, left ventricle; MPP, multipoint pacing; RV, right ventricle; SPP, single-point pacing.

Figure 5 Biventricular activation times using electrocardiographic imaging showing no significant differences among the 3 groups. Box plot showing the $\Delta$ biventricular activation time (shortening compared with baseline) at each of the three pacing configurations: FOI, MPP, and FOI+MPP. The median activation time is indicated by the black line inside the box. FOI, fusion-optimized intervals; MPP, multipoint pacing; SPP, single-point pacing.

Figure 6 Scatter plot showing a positive linear correlation between the paced-QRS duration (via electrocardiography) and biventricular activation time (measured by ECGI) at each pacing configuration: FOI, MPP, and FOI+MPP. FOI, fusion-optimized intervals; MPP, multipoint pacing.

\section{Supplementary Material}

Figure S1. Quadripolar left ventricular lead (Quartet, model 1458Q, Abbott). We first chose one of the two distal electrodes (D1 or M2). Subsequently, we chose one of the two proximal electrodes (M3 or P4), prioritizing the maximum distance between LV1 and LV2: D1/P4 > D1/M3 or M2/P4.

Figure S2 Description of the fusion-optimized atrioventricular (AV) interval method. During atrial sensing, the AV interval was progressively shortened by $20 \mathrm{~ms}$ during left ventricular (LV) pacing alone, from intrinsic conduction with a long AV interval (second left) to only LV activation with a short AV interval (right). The 
AV interval that provided the narrowest QRS was selected and considered the fusion-optimized AV interval (AV FOI). QRSd, QRS duration.

Figure S3 Evaluation of cardiac asynchrony at several levels using echocardiography. A Left intraventricular: septal flash on M-mode imaging (yellow arrows). B Atrioventricular: diastolic filling ratio (diastolic filling time $[\mathrm{DFT}] / \mathrm{RR}$ interval duration). $\mathbf{C}, \mathbf{D}$ Interventricular: the difference between the left (D, orange ) and right $(C$, blue $)$ ventricular pre-ejection times using pulsed-wave Doppler.

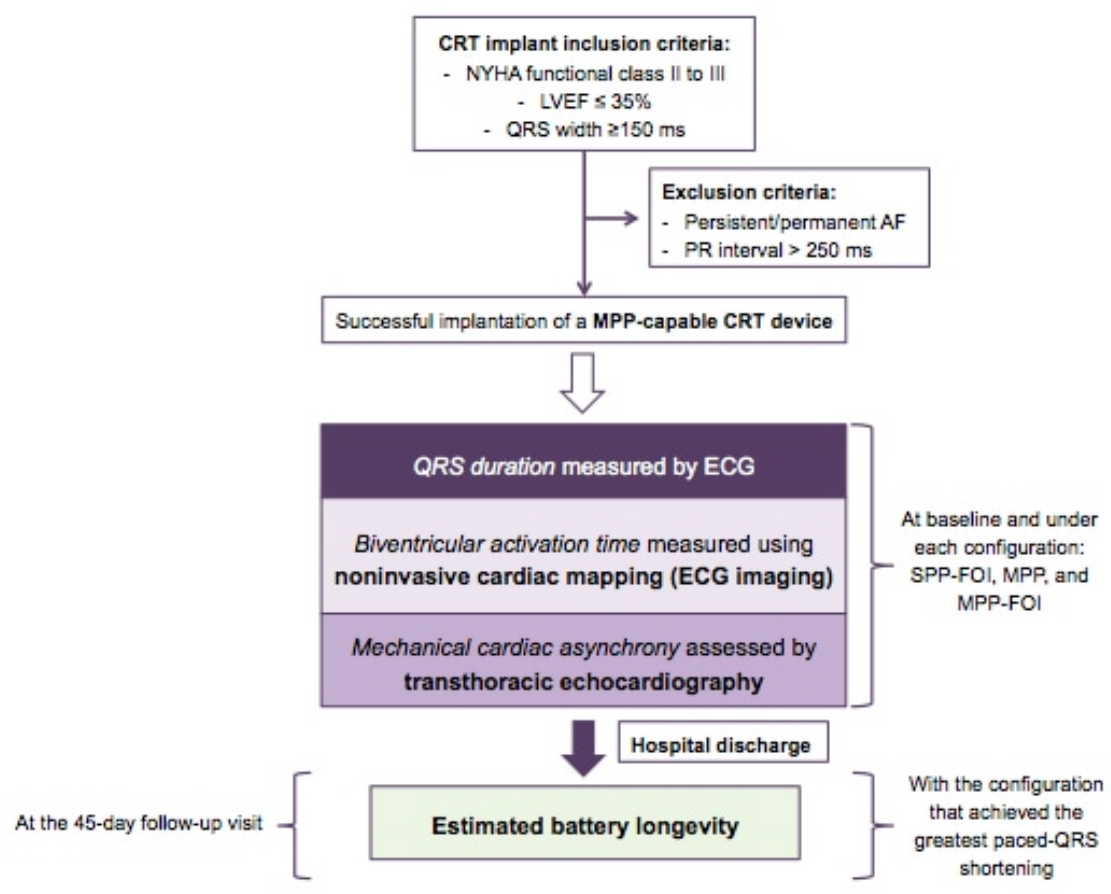



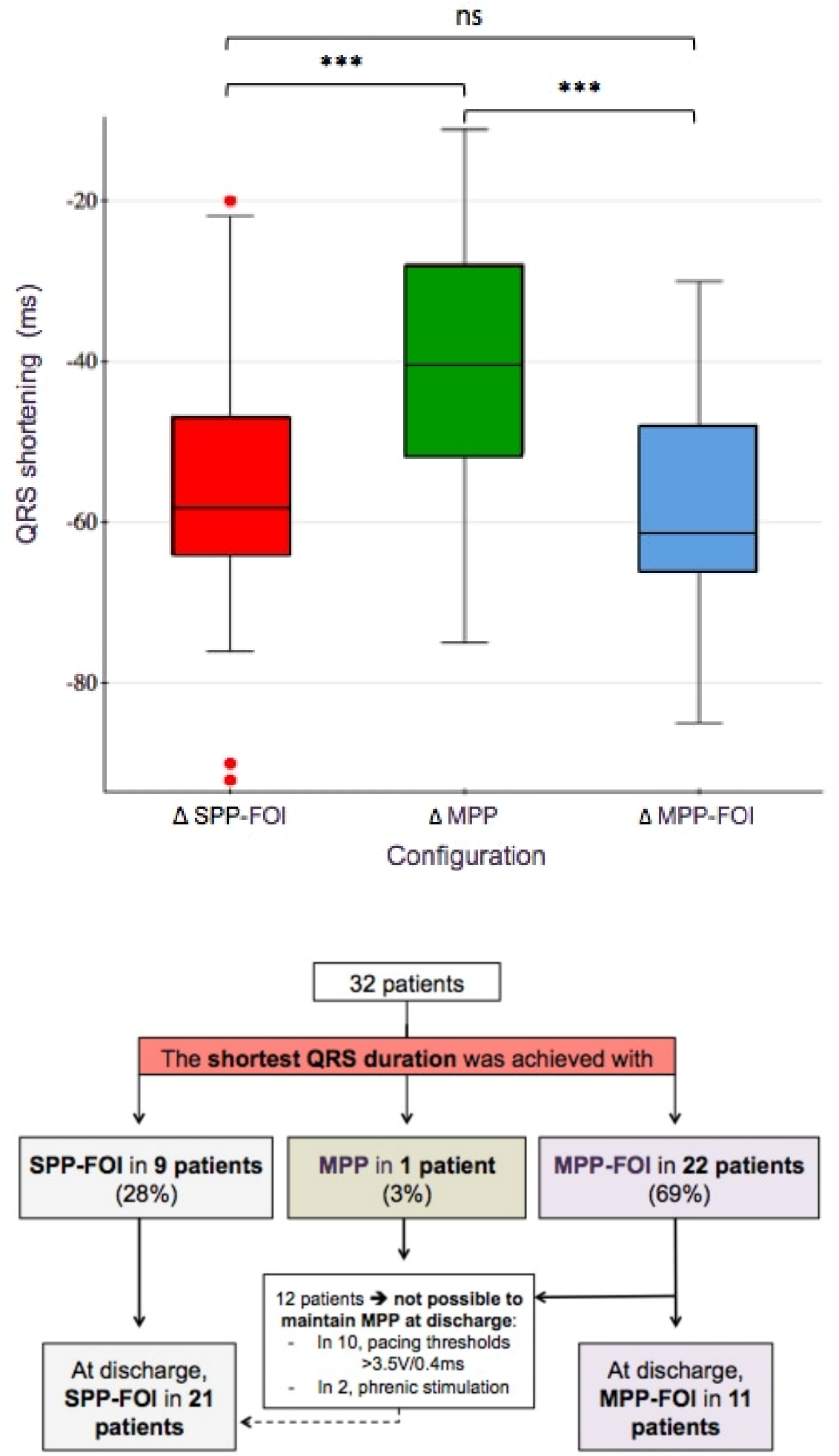


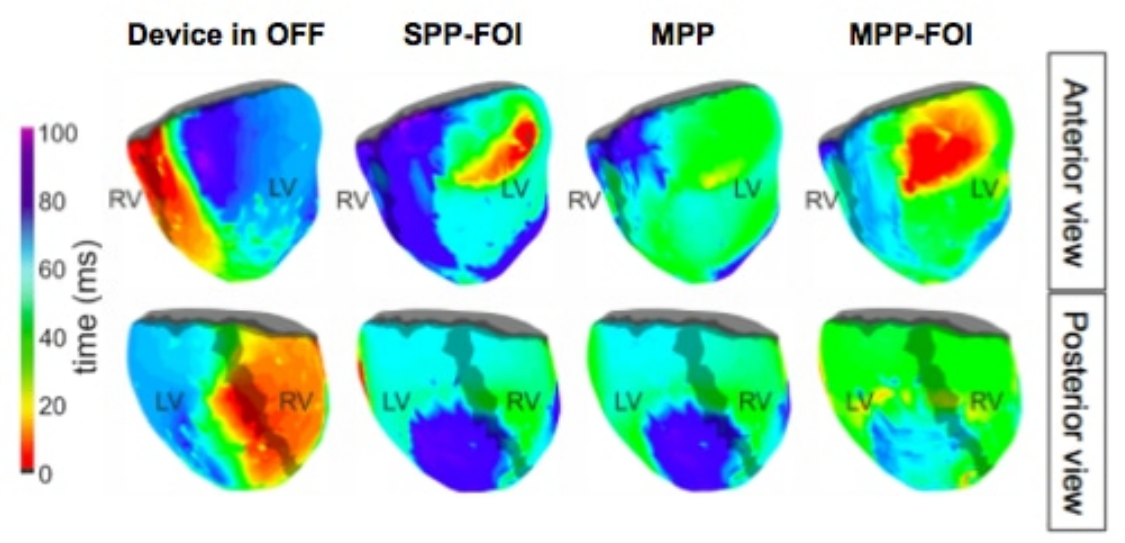

Biventricular activation time

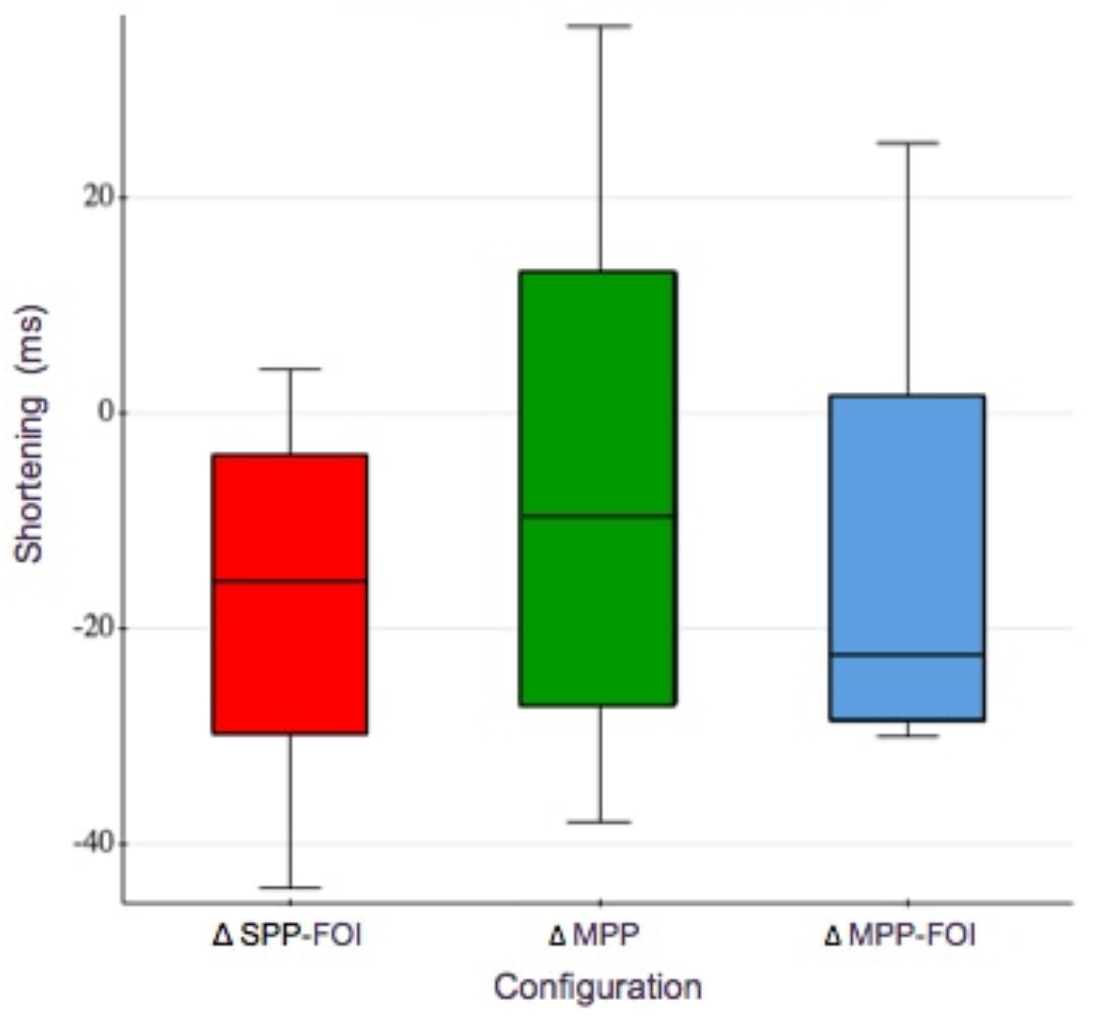




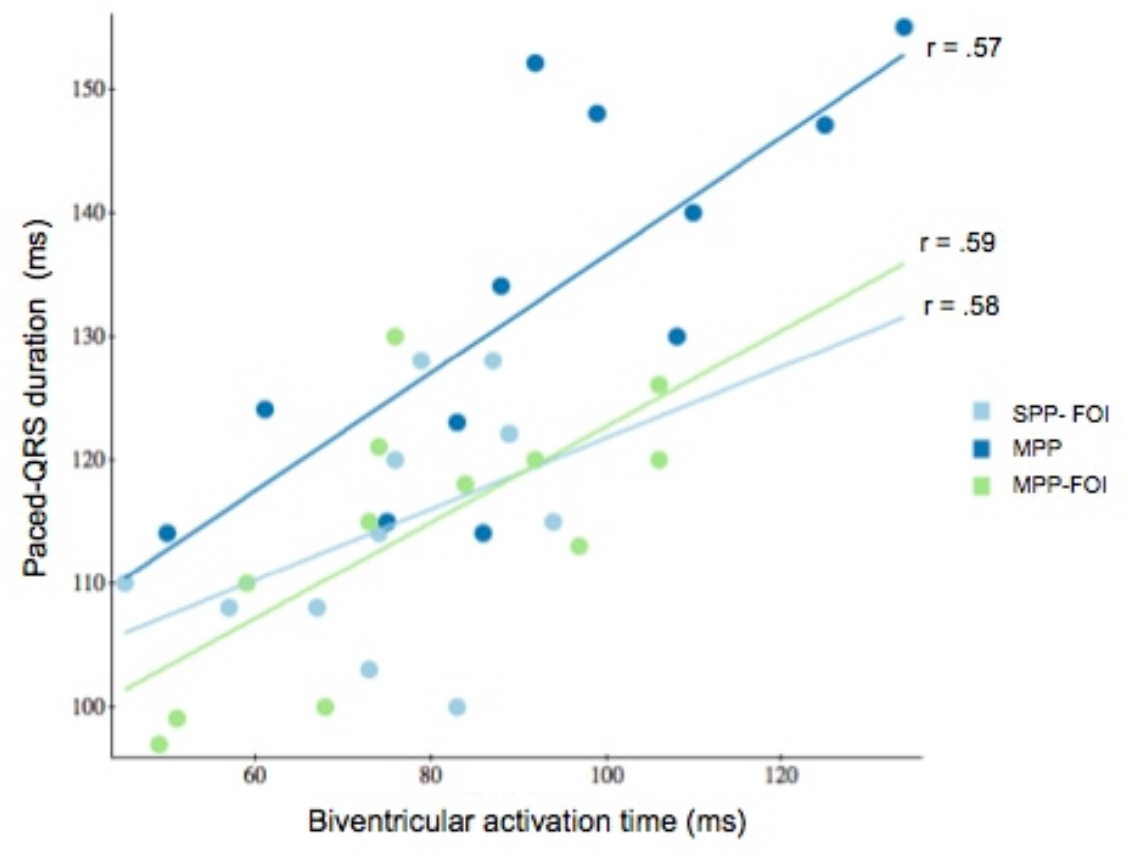

\section{Hosted file}

Table 1.pdf available at https://authorea.com/users/364394/articles/484840-single-pointleft-ventricular-pacing-optimized-by-ecg-results-in-better-resynchronization-thanmultipoint-pacing 